\title{
HIASA: Hybrid Improved Artificial Bee Colony and Simulated Annealing based Attack Detection Algorithm in Mobile Ad-hoc Networks (MANETs)
}

\author{
P.V.S. Siva Prasad and Dr.S. Krishna Mohan Rao
}

\begin{abstract}
A Mobile Ad-hoc Network (MANET) is a persistently self configuring, infrastructure-less network of mobile devices in which every device is wireless, be in motion devoid of constraint and be a router to get across traffic unrelated to its individual use. Each device ought to be organized to persistently maintain the information, mandatory for routing the traffic. Furthermore this is the major confront in constructing a MANET. As of a security design viewpoint, MANETs contain no apparent line of defense; that is to say no integral security. Therefore, the wireless channel is available to both legal network users and malevolent invaders. Consequently the attack investigation is a difficult issue, particularly in MANET where there are only some well-known attacks. Presently, numerous competent routing protocols have been presented for MANET. The majority of these protocols presume a trusted and supportive environment. On the other hand, in the subsistence of malevolent nodes, the networks are susceptible to various types of attacks. In this work a new Hybrid Improved Artificial bee colony and Simulated annealing (HIASA) based attack identification algorithm is proposed to examine various kinds of attacks. The presented HIASA based Attack Detection (HIASA-AD) utilize Artificial Bee Colony (ABC) and Simulated Annealing (SA) algorithm to resolve various types of attacks for instance sybil attacks, wormhole attacks, routing attacks. Fundamental behavior of the nodes, attacks was identified amid source to destination all through Ad hoc On-Demand Distance Vector (AODV) and Dynamic Source Routing (DSR) protocols. An attack could be decomposed into some mixtures of fundamental events. Afterward define taxonomy of anomalous basic events by examining the fundamental security objectives. $A O D V$ and DSR routing protocols are examined in relation to throughput, Packet Delivery Ratio (PDR), and End to End (E2E) Delay. The functioning of AODV is superior to DSR in relation to PDR, throughput, and E2E delay. As the DSR is a reactive routing protocol, it is containing a smaller amount end to end delay as contrast to AODV. These AODV and DSR routing protocols are simulated by Network Simulator-2(NS2) to authorize HIASA-AD algorithm.
\end{abstract}

Index Terms--- Mobile Ad-hoc Network (MANET), Routing Protocols, Attack Detection, Hybrid Improved Artificial Bee Colony and Simulated Annealing (HIASA),

P.V.S. Siva Prasad, M.Tech, Associate Professor, Research Scholar, Rayalaseema University, Andhra Pradesh.E-mail:sivaprasadpvs@gmail.com

Dr.S. Krishna Mohan Rao, M.tech, Ph.D, Principal, Gandhi Institute For Technology(GIFT), Bhubaneswar.E-mail:principal@gift.edu.in

DOI: 10.9756/BIJIEMS.10491
Artificial Bee Colony (ABC), Simulated Annealing (SA) Enhancement, Security, Performance Metrics.

\section{INTRODUCTION}

$\mathrm{M}$ ANETs being a promising technical area is an active area of research and has identified usage in various situations such as disaster relief, military service, emergency processes, and task forces. Granting security to the nodes and their data Communication in such situations is serious. A Mobile Ad hoc Network (MANET) is a self-configuring network which is created without human intervention by a group of mobile nodes with no assist of a permanent infrastructure or central control. Every node is facilitated with a wireless transmitter and receiver that let it to commune with some other nodes in its radio communiqué range. So as to send out a packet to a node that is beyond its radio range, the assistance of some other nodes in the network is needed; this is called multi-hop communication. Consequently, every node has to play as both a host and a router at the same time. The network topology often alters because of the mobility of nodes since they travel inside, travel into, or leave from the network [1-2].

MANET integrates routing functionality into mobile nodes with the intention that they turn out to be able to transmit packets in support of other nodes and therefore efficiently turn out to be the infrastructure. Giving numerous routing paths among any source-destination couple of nodes has confirmed to be extremely helpful in the perspective of wired networks [3].

Security continues a main confront for these networks because of their characteristics of open medium, vigorously varying topologies, dependence on cooperative methods, nonappearance of centralized monitoring points, and be short of clear lines of defence [4]. For instance, lot of the routing protocols presented for MANETs presume that every node in the network is obliging and not malevolent [5]. So, merely one compromised node could be the reason for the collapse of the complete network. Both passive and active attacks are there in MANETs. In case of passive attacks, packets comprising secret information may be snooped that infringes confidentiality. Active attacks, involves infusing packets to untrue targets in the network, altering the contents of packets, deleting packets, and masquerade as other nodes infringe accessibility, reliability, authentication, and non-denial. Proactive techniques for example cryptography and 
authentication [6] were first taken into account, and lot of methods have been presented and implemented.

Lot of the routing protocols for MANETs are therefore susceptible to different kinds of attacks. Security is a major viewpoint in the setting up of MANETs. Literature is copious in describing protocol extensions to give more secure MANET communications. As well lot of methods has been presented to recognize diverse kinds of network attacks, for instance the wormhole attack. On the other hand, all these security solutions are developed for particular routing protocols [7]. In the nonexistence of generic security architecture, nodes from diverse MANET domains could not collaborate and get profit from security benefits crossways the whole network, for instance secured inter-domain routing, and so on. Most confronts arise with implementing these networks [8]. Nevertheless, these applications are inadequate. If we have the capability to identify the attack one time it reaches the network, we could come off it from doing any harm to the system or else any data. There are two situations of MANET; they are diverse from one another in a lot of ways:

- The mobile devices necessitate working merely in a secure and gracious environment where the networking conditions are knowable [9]. Therefore no particular security constraints are required.

- The devices work in an enormously aggressive and challenging environment, wherein the security of the communication and accessibility and operation the network both extremely susceptible devoid of strong security.

Reactive routing protocols could radically lessen routing overhead as they do not require looking for and sustaining the routes where there is no data traffic [10]. The major problems are more latency time in route discovering and extreme flooding could bring about network clogging. Single path routing is dependent upon single route establishment amid source and destination. In this routing, the packet is sent to the target by a single route [11]. Multipath routing provides the option to the source to select the path amid source and target node by considering benefit of the connectivity idleness of the underlined network. Routing tables could be statically allotted or vigorously constructed and updated. In case of static routing systems, the path to transmit traffic amid pairs of nodes is decided devoid of considering the present network state. One time described the paths to be utilized for every source and destination pair, data is constantly transmitted all along these paths. In dynamic (or adaptive) routing system, the routing tables are vigorously updated in relation to the present traffic events and topological changes (for example Link/Node failures, Link/Node addition/removal). Generally, the weakness of these methodologies is that they bring in a heavy traffic load to exchange that is more costly on the subject of the bandwidth-restraint for MANET nodes with inadequate computational capabilities.

Swarm Intelligence (SI) is the collective conduct of self structured systems, decentralized, natural or non-natural [1213]. The motivation frequently originates from nature particularly biological systems. Principle of SI is a multi agent system which contains self-structured conduct which exhibits certain intelligent conduct. They give the subsequent description of swarm intelligence: "Swarm Intelligence (SI) is the collective conduct of (simple) agent interacting locally with their environment root to coherent functional global patterns to come forward". Nature's self-organizing systems, for instance termite hills, insect societies, bird flocks bee colonies, and fish schools, give specifically these characteristics and therefore have been a basis of motivation for the development of lot of routing methods for MANETs.

Designing an effective Intrusion Detection System (IDS), and other security methods, needs an in depth perceptive of threat models and adversaries' attack abilities. MANET utilizes a TCP/IP stack; a lot of familiar attacks could be used to MANET however previous security measures in wired networks could take in hand these attacks. Alternatively, certain protocols, specially routing protocols, are MANET specific. Only a few attack cases of the routing protocols were well analyzed. It goes behind that traditional attack analysis could not work efficiently. Hybrid Improved Artificial bee colony and Simulated annealing (HIASA) based Attack Detection (AD) algorithm is functioned dependent upon the theory of reactive routing protocols(AODV, DSR) in an on demand means for MANETs. Route detection and route maintenance are the stages of HIASA-AD. The sender transmits forward bees in the route detection stage and the bees are passed on by every midway node till it arrives at the destination node. It is simple to analyze the AODV and DSR routing protocols conduct very exactly from the viewpoint of a single node. Specially, analyze the fundamental routing behavior in MANET.

\section{RELATED WORK}

Various protocols for routing have been presented up to now with the intention of identifying a hopeful method that is valid in all areas of MANETs. Certain works in the literature is described along these lines:

Security is an important need in MANETs to give confined communication among mobile nodes. Because of exclusive features of MANETS, it generates a lot of significant confronts to its security design. MANETs are liable to diverse attacks, black hole, is one among the probable attacks. Black hole is a kind of attack in which a malevolent node publicizes itself as containing the shortest path to every node in the location by forwarding false route reply. As a result, the malevolent node could divest the traffic from the source node. It could be utilized as a denial-of-service attack wherein it could drop the packets afterward. Raj and Swadas [14] introduces a novel Detection, Prevention and Reactive AODV (DPRAODV) to protect security risks of black hole by alerting some other nodes in the network of the event. The simulation outcomes in ns2 (ver- 2.33) exhibit that this DPRAODV protocol thwarts black hole attack however as well enhances the functioning of (normal) AODV in existence of black hole attack.

Tsou et al [15] introduced a DSR based secure routing protocol, known as BDSR (Baited-Black-hole DSR) that could identify and evade the black hole attack. BDSR utilizes the conception of transmitting bait and attract black hole to 
respond the false routing information. So, it could attain proactive detection and track the route to accurate location of the available black hole in the preliminary stage. That could lessen the chance of experience black hole attack subsequent to the setting up of the route. They presume that when there is a noteworthy drop in packet delivery ratio, an alarm would be forwarded by the destination node to the source to activate the discovery method once more that could attain the ability of maintenance and instantly reactive response.

Wang et al [16] propose a technique for including hash based into REAct system. Consider the behavioral proof could enclose both information from data traffic and transmitting paths. Both of them consider assisting source node to identify malevolent node. The above two papers describe that the discovery approach activate merely while the destination node identifies noteworthy drop in packet delivery ratio. Alternatively, the overhead of the methods imply creates while the path subsist malevolent node and the malevolent node starts to attack. On the other hand, these techniques till create MANETs to experience packets loss in preliminary stage and effect some harm to network. This type of discovery method is in reactive methodology.

AlMobaideen [17] introduces a novel Stability-based Partially Disjoint AOMDV (SPDA) protocol that is an alteration of the AOMDV protocol. SPDA identifies partly disjoint paths dependent upon links constancy. The plan is that recognizing partly disjoints paths that are very constant compared to other maximally disjoint ones can amplify paths life span. This consecutively progresses MANET performance in relation to, routing packets overhead, delay and the network throughput. Outcomes of certain experiments, which we have carried out, utilizing Golomosim simulator package, exhibit an enhancement in the performance of the presented protocol on the subject of the considered performance metrics.

Di Caro et al [18] explain a novel AntHocNet, a methodology for routing in mobile ad hoc networks. It is a hybrid technique that joins reactive path setup in the company of proactive path probing, maintenance and enhancement. The technique is dependent upon the nature-enthused ant colony enhancement structure. Paths are well-read by guided Monte Carlo sampling utilizing ant-like agents communicating in a stigmergic means. In a widespread set of simulation experiments, contrast AntHocNet with AODV, a reference algorithm in the area. The outcomes prove that the AntHocNet algorithm could do better than AODV on diverse evaluation criterion AntHocNet's performance improvement is noticeable over a wide range of probable network situations, and enlarges for larger, sparser and more mobile networks.

Rahmatizadeh et al [19] presented a novel agent-based routing algorithm, which is dependent upon both ant and bee colony enhancement methods called the Ant-Bee Routing (ABR) algorithm. In this method, the primary AntNet Routing (ANR) algorithm is changed where certain intellectual characteristics of bees are incorporated to improve the performance of the ANR algorithm. Experiments with the ABR algorithm are developed by the OMNET++ software platform through NTTNET network topology. Outcomes proved that the performance of ABR algorithm is very better compared to the ANR algorithm with decrease in packets delay. The ABR is as well appropriate in dynamic environment.

Mazhar and Farooq [20] examine the security susceptibilities of BeeAdHoc, a familiar power aware, Bio/ Nature-enthused routing protocol. The study undoubtedly expresses that the malevolent nodes in an untrusted MANET, where BeeAdHoc is utilized for routing, could considerably disturb the usual routing behavior. Afterward present a security framework, BeeSec, for BeeAdHoc which facilitates it to counteract the diverse kinds of threats. They as well developed a pragmatic framework, embedded into a familiar simulator, ns-2, to methodically legalize the operational security of BeeSec. A remarkable result of the presented is that BeeSec, even with noteworthy overhead of the security framework, attains enhanced performance while matched up with state-of-the-art, non-secure, classical routing protocols AODV and DSR.

Mazhar and Farooq [21] introduce a dendritic cell based distributed misbehaviour identification system, BeeAIS-DC, for a Bio/Nature enthused MANET routing protocol, BeeAdHoc. Our presented enthuses from the danger theory and models the function and conduct of dendritic cells to identify the existence or nonexistence of danger and gives a tolerogenic response. The presented detection system is executed in a renowned ns-2 simulator. The outcomes point to that our detection system not just facilitates BeeAIS-DC to vigorously acclimatize its detector set to provide for a altering self due to mobility of nodes, but in addition is vigorous enough to give considerably small false positives while matched up with self/non-self based AIS. Furthermore, the danger theory associated overhead of BeeAIS-DC is minimum, and consequently, it does not disgrace traditional performance metrics of BeeAdHoc. This conduct is very important for battery/bandwidth restrained mobile nodes.

Barani and Abadi [22] propose a dynamic hybrid technique dependent upon the Artificial Bee Colony (ABC) and negative selection (NS) methods, known as BeeID, for intrusion detection in AODV-based MANETs. The method comprises three stages: training, detection, and updating. In the training stage, a niching $\mathrm{ABC}$ algorithm, named NicheNABC, runs a negative selection technique several times to produce a set of mature negative detectors to cover up the non-self space. In the detection stage, mature negative detectors are utilized to distinguish among usual and malevolent network activities. In the updating stage, the set of mature negative detectors is brought up to date by one among two techniques of half-done updating or total updating. Afterward utilize the Monte Carlo integration to guesstimate the quantity of the nonself-space covered by negative detectors and to find out when the total updating should be carried out. The outcomes show the efficiency of BeeID for identifying numerous kinds of routing attacks on AODVbased MANETs simulated utilizing the NS2 simulator. The experimental outcomes prove that BeeID could attain a superior trade-off attack detection rate and false-alarm rate while matched up with other dynamic techniques formerly stated in the literature. 
In the current work presents a modified bio-inspired routing protocol known as Secure Artificial Bee ColonyAODV (SABC-AODV) [23] for protected routing of data in MANET. It utilizes the method of artificial bee Colony algorithm and AODV protocol. The presented method is proficient as it utilizes a new mixture of group signature and ID-based encryption for route detection. The simulation outcomes are better for improved packet delivery ratio and decreased end to end delay in MANET and as well alleviate the Black hole attack.

\section{OPTIMAL INTRUSION DETECTION SYSTEM FOR MANET}

Constructing an effective Intrusion Detection System (IDS), likewise rest of the security mechanisms, demands a wider knowledge of threat models and adversaries' attack capabilities. MANET utilizes a TCP/IP stack, much wellestablished intrusion can be enforced to MANET but current security measures in equipped networks can explain these intrusions. Further, few protocols, specifically routing protocols, are MANET particular. Very few intrusion cases of these routing protocols have been well examined. It pursues the conventional intrusion examinations, which doesn't work efficiently. Hybrid Improved Artificial bee colony and Simulated annealing (HIASA) based Attack Detection (AD) algorithm depends on the standard of reactive routing protocols(AODV, DSR) in a demanded way for MANETs. The main target of HIASA-AD is to examine and identify the intrusions which minimize the routing overhead for reactive routing protocols (AODV, DSR). It is broadly flexible, effective and extensible. It won't utilize any HELLO message to absolutely identify its neighbors. When a packet reaches at node, the node audit it to verify if the routing details is available for destination in its routing table. Route discovery and route maintenance are the phases of HIASA-AD. The sender transmits forward bees in identifying the route phase and the bee communicate with every intermediate node till it attains the destination node. It is simpler to examine the AODV and DSR routing protocols nature more precisely from the outlook of a single node. Especially, examine the basic routing behavior in MANET.

\section{A. Routing Protocols}

It is a reactive protocol that generates a way on-demand by utilizing the source routing protocol i.e. it demands an entire sequence of paths has to be developed among the source and destination nodes in order to broadcast the packets and every packet pursue the similar path. The bigger inspiration of this protocol restricts the bandwidth by eliminating the periodic table renovation and huge convergence time.

\section{a. Dynamic Source Routing (DSR) Protocol}

The emphasizing case to this DSR protocol [24] is it will downpour route information in the network to develop a route and it has two procedures: Route Discovery and Route Maintenance.

Route Discovery: According to an on-demand routing protocol, it search for the routing at the time of packet broadcast. During the first phase, transmitting node look for its route cache to check whether there avails an appropriate destination and then the node begins its transmission to the destination node and the route identifies the process end here. If destination address isn't mentioned then the node transmits the route request packet to attain the destination. When the destination node receives the data, it restores the established path to the source node.

Route Maintenance: It is a process of transmitting the information by a node to remaining nodes details in the network or failure of the node in a network. It gives a recent identification of nodes or link failure, because wireless networks uses hop-to-hop acknowledges.

\section{b. Ad Hoc On-Demand Distance Vector (AODV) Routing Protocol}

The AODV is constituently a distance vector routing protocol [25] that has been improved for ad-hoc wireless networks. It is an on demand or reactive protocol, as it identifies the way only on demand. AODV obtain fundamental route development and maintenance mechanisms from the DSR protocol and hop-to hop routing vectors from the Dynamic Source Routing (DSR) protocol. In order to eliminate the issue of routing loops, AODV creates considerable utilization of sequence numbers in control packets. When a source node intends to interact with a destination node whose route is unknown, it transmits a Route Request packet (RREQ). Every RREQ has an Identifier (ID), source and destination node IP addresses, and sequence numbers together with a hop count and control flags. The ID field specifically finds the RREQ; the sequence numbers points the novelty of the control packets and the hop-count controls the number of nodes among the source and the destination. Every recipient of the RREQ that doesn't have an idea about the source IP and RREQ ID pair or does not have a fresher (with larger sequence number) route to the destination retransmits the exact packet subsequently increasing the hopcount. An intermediate node creates and continues a Reverse Route to the source node for a specific interval of time. When the RREQ attains its destination node or any node which has a fresh way to the terminus, a Route Reply packet (RREP) is created and unicast return to the initiating point of the RREQ. Every RREP has the destination sequence number, the source and the destination IP addresses, route lifetime, and a hop count and control flags. Every intermediary node that obtains the RREP increments the hop-count, develops a Forward Route to the source of the packet, and broadcasts the packet through the Reverse Route. To continue connectivity details, every node implementing AODV can utilize link layer feedback or periodic HELLO packets in order to identify the link breakages to nodes, which is assumed as its immediate neighbours. In case a link break is identified for a next hop of an active route, a Route Error packet (RERR) is given to its active neighbours that utilize that specific route. While utilizing the ODV in a network with multi-radio nodes, every RREQ transmits on entire interfaces. In order to prevent broadcast storms, a random delay is included in broadcasting every RREQ. Intermediate nodes with one or more interfaces works on a shared channel, it obtains the RREQ and generates a Reverse Route that points towards the source node. If the 
RREQ is redundant, it is easily discarded. The RREQ which is obtained $1^{\text {st }}$ by the destination or any intermediary node is chosen and remaining RREQs will be dropped. The RREP is created in response to the chosen RREQ, and it is given back to the source node on the existing Reverse Route.

The merits of this protocols are 1) Knowledge of available of different paths that assist to identify other path if failure occurs in the node or link. 2) It prevents routing loops and 3) less preservation overhead cost as it is an on-demand routing protocol. Further, the demerits are 1) if the route length is long while receiving delay for identifying the route; it is not adequate in position like the battle field. 2) It isn't applicable for huge nodes when speed differs and 3) it generates higher messaging overhead in busy schedule. Among these routing protocols route discovery and route maintenance is considered as extensive task. Since, during this phase, the broadcasting node looks for a route cache to check whether there avails an appropriate terminus and if it exists, the node begins broadcasting to the destination node and the route discovery process end here. At the time of this process, security issues became a significant problem.

\section{B. Network Model}

Let us assume $G=(V, E)$ be a connected graph with $n=|V|$ nodes [26]. The bee system can be utilized to identify the shortest path among a source node $\mathrm{v}_{s}$ to destination node $\mathrm{v}_{d}$ on the graph $\mathrm{G}$. The path length is provided by node's count on the link. Every edge $e(i, j) \in E$ of the graph connecting the nodes $v_{i}$ and $v_{j}$ has a variable $\mathrm{f}_{\mathrm{ij}}$ (artificial food source), which is changed by an adjusted bees when they visit the node. The food source $f_{i j}$ is an implication of the utilization of this edge. An adaptive bee located in node $v_{i} u$ tilizes the food source $\mathrm{f}_{\mathrm{ij}}$ of node $\mathrm{v}_{j} \in \mathrm{N}_{i}$ to estimate the probability of node $\mathrm{v}_{j}$ as next hop.

\section{Hybrid Improved Artificial Bee Colony and Simulated Annealing (HIASA) based Attack Detection(AD) Algorithm}

Hybrid Improved Artificial bee colony and Simulated annealing (HIASA) depends on the principles of reactive technique in an on demand way for MANETs. It is greatly flexible, effective and extensible. It doesn't utilize the HELLO message to absolutely identify the neighbors. When the packets reach the nodes, it validates the routing details, whether it is available for destination in its routing table. Route discovery and route maintenance are the phases of HIASA. The sender transmits the forward bees in the route discovery phase and the ant is communicated by every intermediate node till it attains the destination. The adaptive bee can be separated into two sections: Forward Adaptive Bees (FAB) and Backward Adaptive Bees (BAB). It is utilized to generate the new routing path. FAB is answerable for developing the honey bee food source path to the initial node and $\mathrm{BAB}$ is answerable for developing the honey bee path to the terminus node. At the time of the journey FAB from source to destination, when the $\mathrm{FAB}$ is obtained at the intermediate nodes for the very first time, the recipient node obtaining a FAB for the very first time constructs a record of following parameters i.e., source address, destination address, next hop, hop to hop count distance, node position, data packet size, packets information, honey bee value, attacks analysis threshold values in its routing table. During this time, when the FAB attains the destination node, it is processed in a special manner. The destination node drew-out the details from the $\mathrm{FAB}$ and then eradicates the FAB. After that a $\mathrm{BAB}$ is generated at the terminus node and return back to the initial node on the reverse path that was followed the FAB. Similarly, the route is developed among the source and destination and data packets can be sent. As shown in Figure 1. The initial node generates a Forward Adaptive Bees (FAB) and sends these bees are designed to detect the route to its neighbours nodes. Utilizing probabilistic decision $p_{i}$ it confirms the next hop node and forwards these bees through entire next hop nodes till it attains the destination.

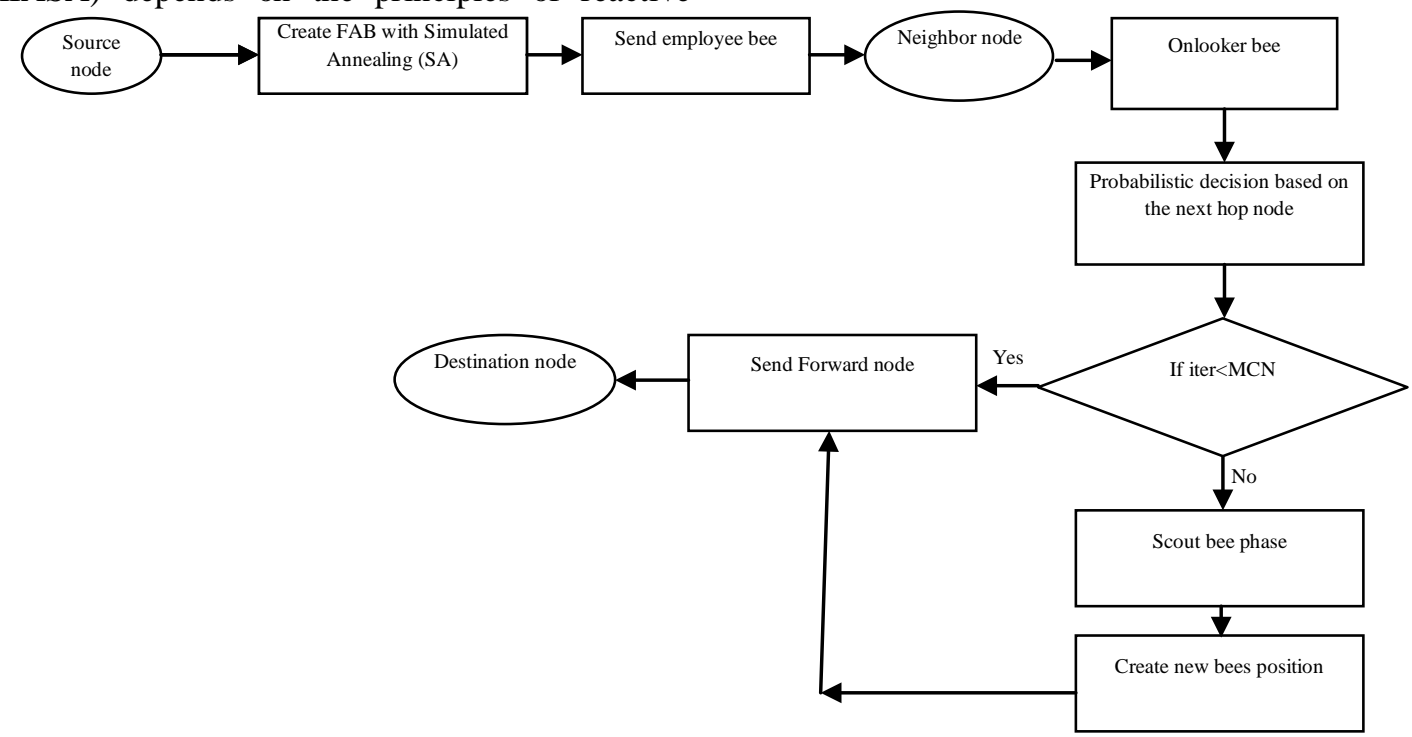

Figure 1: Transmission of Forward Adaptive Bees (FAB) in HIASA based AD algorithm 
As shown in Figure 2. The destination node generates a Backward Adaptive Bees (BAB) and sends these bees in the similar route traces created by the forward ant via the intermediate nodes till it attains the source node

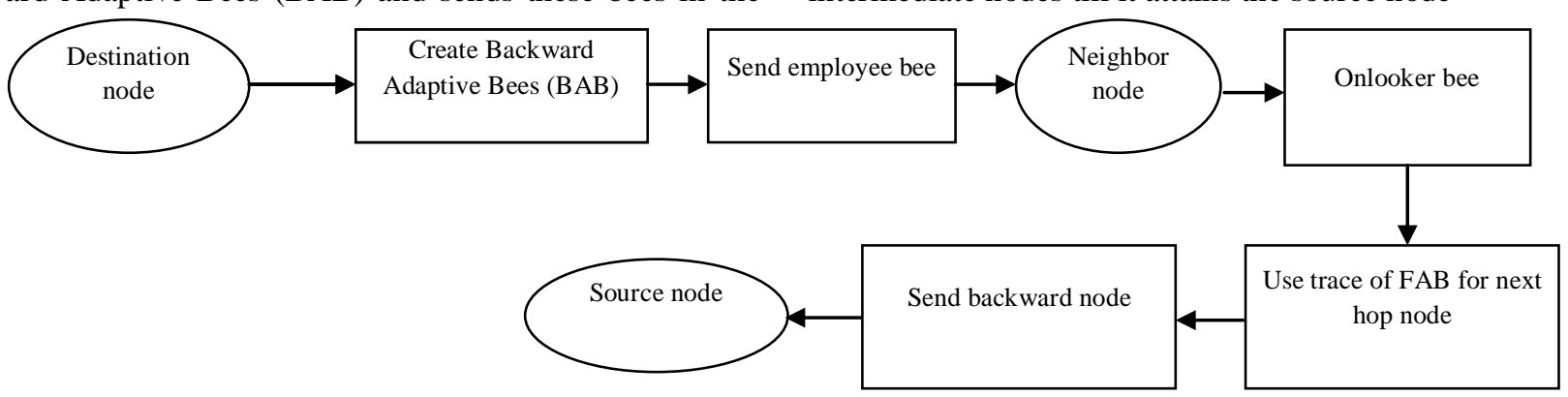

Figure 2: Transmission of Backward Adaptive Bees (BAB) in HIASA based AD Algorithm

Through this broadcasting manner of MANET usually associates three elements or targets: routing messages, data packets and routing table (or routing cache) opening. They study of the fundamental MANET routing intrusions which are observed in the articles [27-30]. By distinguishing them (given in Table 1), identify the match with the definitions of anomalous basic task. Mention every intrusion with a specific name and deliberately a suffix letter. For instance, "Route Flooding (S)" is a flooding attack of routing messages that utilizes a specific source address. Furthermore, it assumes a number of difficult intrusion scenarios that has a sequence of anomalous basic events

Table 1: Basic MANET Attacks, where Suffix Letters Stand for Different Attack Variations: R, S, D Stand for Randomness, Source only and Destination only, Respectively. Other Letters Include M (maximal value), F (failure), Y (reply), I (invalid) and $\mathrm{N}$ (new)

\begin{tabular}{|c|c|c|}
\hline Attacks & Attack Description & equivalent Anomalous Basic Events \\
\hline Active Reply & A Route Reply is actively forged with no related arriving Route Request information. & \\
\hline False Reply & $\begin{array}{l}\text { A Route Reply is forged for a Route Request message even though the node is not supposed } \\
\text { to reply. }\end{array}$ & Fabrication of routing Messages \\
\hline Route Drop (R) & Drop Routing packets. (R) Denotes a random selection of source and destination addresses. & \multirow{3}{*}{ Interruption of Routing Messages } \\
\hline Route Drop (S) & $\begin{array}{l}\text { A fixed percentage of routing packets with a specific source and destination address are } \\
\text { dropped. (S) stands for source address. }\end{array}$ & \\
\hline Route Drop (D) & $\begin{array}{l}\text { A fixed percentage of routing packets with a specific destination address are dropped. (D) } \\
\text { stands for destination address. }\end{array}$ & \\
\hline $\begin{array}{l}\text { Modify sequence } \\
\text { (R) }\end{array}$ & Modify the destination’s sequence number randomly. (R) stands for randomness. & \multirow{3}{*}{ Modification of Routing Messages } \\
\hline $\begin{array}{l}\text { Modify sequence } \\
\text { (M) }\end{array}$ & $\begin{array}{l}\text { Increase the destination's sequence number to the largest allowed number. (M) stands for the } \\
\text { maximal value. }\end{array}$ & \\
\hline Modify Hop & Change the hop count to a smaller value. & \\
\hline Rushing (F) & $\begin{array}{l}\text { Shorten the waiting time to send a Route Reply after a Route Request is received. (Y) stands } \\
\text { for reply. }\end{array}$ & \multirow{2}{*}{ Rushing of Routing Message } \\
\hline Rushing (Y) & $\begin{array}{l}\text { Shorten the waiting time to send a Route Reply after a route Request is received. (Y) stands } \\
\text { for reply. }\end{array}$ & \\
\hline Route Flooding (R) & Flood with both source and destination addresses randomized. & \multirow{3}{*}{ Flooding of Routing Messages } \\
\hline Route Flooding (S) & Fold with both source and source address and random destination addresses. & \\
\hline Route Flooding (D) & Flood to a single destination with random source addresses. & \\
\hline Data Drop $(\mathrm{R}|\mathrm{S}| \mathrm{D})$ & Similar to Route Drop (R), Route Drop (S), Or Route Drop (D), but using data packets. & Interruption of data Packets \\
\hline $\begin{array}{ll}\text { Data } & \text { Flooding } \\
(\mathrm{R}|\mathrm{S}| \mathrm{D}) & \\
\end{array}$ & $\begin{array}{l}\text { Similar to Root Flooding (R), Root Flooding (S), or Root Flooding (D), but using data } \\
\text { packets. }\end{array}$ & Flooding of data Packets \\
\hline Add Route (I) & An invalid route entry is randomly selected and validated. (I) stands for invalid. & \multirow{2}{*}{ Add Route of Routing Table Entries } \\
\hline Add Route $(\mathrm{N})$ & A route entry is added directly with random destination address. $(\mathrm{N})$ stands for new. & \\
\hline Delete Route & A random valid route is invalidated. & Delete Route of Routing Table Entries \\
\hline $\begin{array}{ll}\text { Change } & \text { Sequence } \\
(\mathrm{R} \mid \mathrm{M}) & \\
\end{array}$ & $\begin{array}{l}\text { Similar to Modify Sequence attacks but the sequence number is changed directly on the } \\
\text { routing table. }\end{array}$ & \multirow[t]{2}{*}{$\begin{array}{l}\text { Change Route Cost of Routing Table } \\
\text { Entries }\end{array}$} \\
\hline Change Hop & Similar to Modify Hop,, but the hop count is changed directly on the routing table. & \\
\hline Overflow Table & Add excessive routes to overflow the routing table. & $\begin{array}{l}\text { Routing Table Overflow of Routing } \\
\text { Table Entries. }\end{array}$ \\
\hline
\end{tabular}

Worm hole attack: This intrusion basically works in two phases. In the first phase, the wormhole nodes get themselves engaged in various routes. In the second phase, these malignant nodes begin manipulating the packets when they attains. These nodes can disorganize functionality of the network in various ways. For instance, the nodes can astonish the protocols that rely on the node position or geographic closeness, or the conspiring nodes may deliver the data packets to and fro to another in case of virtual tunnel so as to cripple the battery of other intermediate nodes. Wormhole nodes can discard, change or send the data to a third party for malignant purpose.

Sybil attacks: A node that desires to identify the existence of the Sybil attacker in the network begins by recording the identities of every other node it hears transmitting over a sequence of intervals. An entire record of complete data transmission isn't required. The observation period is indicated as the time bucket, is enough to obtain the probable nature of the normal node including: normal data flow, 
common HELLO and keep-alive messages, and periodic route requests for nodes that have data to send but it doesn't possess existing route to the terminus. The length of this time period is based on the fundamentally applied protocol; in the simulations 40 seconds was enough as it far superior for the period among the routing updates and requests

\section{a. Hybrid Improved Artificial Bee Colony and Simulated Annealing (HIASA)}

The above addressed nature of the intrusion is examined and identified through the utilization of HIASA algorithm, it is brought in for these changes which are Simulated Annealing (SA) initialization, self-adaptive mechanism for employed bees and onlooker bees steps, and chaotic Opposition Based Learning (OBL) for scout bee step and enhance the convergence performance of standard ABC algorithm according to the benchmark optimization issues. Population initialization is a crucial stage in SI algorithms since it affects the convergence speeds and the quality of the final solution. If no details are available, then random initialization is generally utilized method for creating a basic population. Local search method is commonly integrated with the population according to the search algorithm. Then a SA is brought-in as a local search method and which is correlated with ABC algorithm. The initial search algorithm investigates the most hopeful search space regions while the exploitation's capability is enhanced via SA through auditing the surroundings of basic solutions. In the past, the insect behaviours of animals and insects that present significant properties of SA have been examined in different investigations.

\section{Initialization of Bees Using SA}

The process of annealing [31] can be imitated with the Metropolis algorithm, which depends on the Monte Carlo techniques. Here, SA is enforced to create a solution to initial population in IABC algorithm. To enforce the SA with initialization purpose demands the following procedure:

- The successor function returns a close neighboring nodes nature in the MANET model. This will proceed as the "disturbance" for the fragments of the system.

- An objective function or fitness functions to initialization of number of paths rely on the existing MANET network model. This fitness function wills deliberate as the energy of the system. The search is beginning with a randomized MANET network model with their routes. In a polling loop need to navigate to neighboring nodes always obtain the navigation that reduces the energy while only obtaining the bad progress appropriately to a likelihood of dispersion is helpless on the "temperature" of the system.

- Reduce the temperature gradually; accepting less random initialization of bees for attack detection every temperature level till the lowest temperature of the algorithm becomes a greedy hill-climbing algorithm.

- The distribution utilized to agree and obtain a bad progress by utilizing Boltzman distribution

$$
\begin{gathered}
P(\gamma)=\frac{e^{\frac{-E_{\gamma}}{T}}}{Z(T)} \\
Z(T)=\sum_{\gamma^{\prime}} e^{\frac{-E_{\gamma^{\prime}}}{T}}
\end{gathered}
$$

Where $\gamma$ the existing configuration of the system is, $E_{\gamma}$ is the energy accompanied with it, and $\mathrm{Z}$ is normalization constant. Initialization for proposed HIASA using SA is measured as shown in Eq. (3).

$$
X_{i j}^{t+1}=X_{i j}^{t}+\alpha \times S A\left(\gamma_{\text {new }}\right)
$$

where $i \in\{1,2, \ldots S N\}$ and $j \in\{1, \ldots D\}$ are randomly chosen route from the initial to the terminus, $\mathrm{t}$ is the iteration number set for $50, \mathrm{U}(0,1)$ - uniformly distribution function, so far initial and terminus nodes are derivate of normal distributions. To accomplish the good performance and resolve the local optima, we brought-in a self-adaptive mechanism to modify the search range accompanied with a cycle number, and then combined it with $\mathrm{DE}$ to enhance the performance of the employed bees. In the standard ABC algorithm, a random perturbation is included to the existing solution to generate the new solution. This random perturbation is weighted by $\phi_{\mathrm{ij}}$ selected from $[-1,1]$ and it is estimated a form of uniform distribution function. A low value of $\phi_{\mathrm{ij}}$ results in small steps is utilized to identify the optimal route and detects attacks, hence accomplishes the convergence slowly. A greater value of $\phi_{i j}$ advances the search, but minimizes the analyzing capability of the perturbation process.

\section{Self-adaptive Mechanism}

Self-adaptive mechanism is utilized to equalize the analyzing capability and the convergence speed of the algorithm for employed bees. The self-adaptive ABC approach, $\phi_{\mathrm{ij}}$ is modified with the cycle number based on the random value termed in the range [0,1] for identifying the optimal number of routes among the source to destination process of employed bee; $\phi_{\mathrm{ij}}$ is defined as

$$
\phi_{i j}=\left\{\begin{array}{l}
-e^{-3 \times \text { cycle } /(25 \times M C N)}, 0 \leq \text { rand } \leq 0.5 \\
e^{-3 \times \text { cycle } /(25 \times M C N)}, 0.5 \leq \text { rand } \leq 1
\end{array}\right.
$$

To raise the speed of the attack detection algorithm , which is linked with the " $\mathrm{DE} / \mathrm{best} / 1$ " mutation scenario with the process of searching food of the new intrusion in the routing generates a new search equation is determined in the equation (6)

$$
\mathrm{v}_{\mathrm{i}, \mathrm{G}}=X_{\text {best }, G}+F \times\left(X_{r_{1}^{i}, G}-X_{r_{2}^{i}, G}\right)
$$

where $\in\{1,2, \ldots S N\}$ is a randomly chosen index, NP is the population number, $\mathrm{G}$ is the generation number, $\mathrm{v}_{\mathrm{i}, \mathrm{G}} \mathrm{is}$ the donor vector, $r_{1}^{i}$ and $r_{2}^{i}$ are random numbers selected from the range $[1, \mathrm{NP}]$.

$$
\mathrm{v}_{\mathrm{i}, \mathrm{j}}=X_{\text {best }, j}+\phi_{i j} \times\left(X_{i j}-X_{k j}\right)
$$

So, to raise the likelihood of the lower fitness attacks and routes to be chosen through changing $\mathrm{P}_{\mathrm{i}}$, brought-in self adaptive mechanism to $\mathrm{P}_{\mathrm{i}}$. The value of $\mathrm{P}_{\mathrm{i}}$ differs with the updated cycle number, it is based on the adding the value of the fitness and max. value of fitness. $P_{\mathrm{i}}$ is given in $\mathrm{Eq}(7)$

$$
\mathrm{P}_{\mathrm{i}}=\exp \frac{-0.15 \text { cycle }}{M C N} \frac{f i t_{i}}{f i t_{\max }}+\left(1-\exp ^{\frac{-0.15 \text { cycle }}{M C N}}\right) \frac{f i t_{i}}{\sum_{n=1}^{S N} f i t_{i}}
$$




$$
\begin{aligned}
& f i t_{i}= \text { (source address, destination address, next hop, hop to hop count distance, } \\
& \text { node position, data packet size, packets information) }
\end{aligned}
$$

The idea of OBL [32] and has been enforced to increase the supported learning and back- propagation learning in neural networks [33]. The primary concept of OBL is to enhance our chance to begin with a closer (fitter) solution by validating the conflicted solution at the same time. Through this way, the closer one to solution (say guess or opposite guess) can be selected as first solution. Based on the probability theory, in $50 \%$ of cases, the guess is a greater distance to the result than conflicted guess; so, in these scenarios, which begins with the conflicted guess can advance the convergence. OBL was brought-in to DE and enhanced the convergence implementation [34]. Hence, to advance the speed of the convergence and to avoid staying on the local result, initialization approach is brought-in which applies chaotic systems [35] and OBL method for scout bees. In this work, a sinusoidal iterator is chosen for chaotic, and its equation is determined as

$$
\operatorname{ch}_{\mathrm{k}, \mathrm{j}}=\sin \left(\pi c h_{k-1, j}\right)
$$

where $h_{k} \in(0,1), k=1,2, \ldots, \operatorname{Max}, j=1,2, \ldots, D$. The initialization population for scout bees is shown as

$$
\mathrm{X}_{\mathrm{k}, \mathrm{j}}=\mathrm{X}_{\min , \mathrm{j}}+\mathrm{ch}_{\mathrm{k}, \mathrm{j}}\left(\mathrm{X}_{\max , \mathrm{j}}-\mathrm{X}_{\min , \mathrm{j}}\right)
$$

and the chaotic OBL equation is shown as

$$
\mathrm{OX}_{\mathrm{ij}}=\mathrm{X}_{\min , \mathrm{j}}+\mathrm{X}_{\max , \mathrm{j}}-\mathrm{X}_{\mathrm{ij}}
$$

where ox refers the opposition-based population.

\section{HIASA based $A D$ algorithm}

1. Establish a network $N=(1, \ldots n)$ with $\mathrm{N}$ number of nodes

2. Specify the properties of network

3. Define the source and the destination node over the network

4. Initialize cycle to 1

5. Place the bee at each node in the network

6. Define the m malicious nodes over the network

7. Compute fitness $f_{i}$ using the properties of the nodes

8. Initialization of new bee at each node in the network by equation (3)

9. Route discovery process: Source node broadcast the RREQ message to neighboring nodes using FAB forward technique and hop count is initialized. It is an agent to establish food source value $\phi_{\mathrm{ij}}$ to the source node by equation (4)

10. Define new DE/best/1" mutation strategy to find new optimal food searching process in equation (5-6)

i) Collecting the neighboring nodes information stored in routing table

ii) Neighboring nodes receive the request then it will check whether the node is destination or not.

- If yes then

- FAB is sent to only that neighbour

- Else

- $\quad$ it's forwarded to all the neighbors.

- A node is receiving a FAB for the first time, will create a record in its routing table and fields such as destination address, next hop and pheromone value.
11. For each FAB (currently in node i)

Do

- Choose the neighbour node, probability value equation (7) will be high that route/neighbour needs to be considered

- $\quad$ Add that node bee value to neighboring routing table with the node, bee value between these nodes until the FAB has reached the destination.

End

12. If the solution does not improve, add 1 to the trail, otherwise, set the trail to 0 .

13. Determine the abandoned solution through the number of limit for the scout, if it exists, and replace it with a new random solution using Eq. (11).

14. The full process is mention above to get repeated until the FAB reaches the destination node.

15. When FANT destroy, it is reaches to the destination and create $\mathrm{BAB}$ send to along the path to the source node. It is an agent that establishes the pheromone value to the destination.

16. Memorize the best solution achieved so far.

17. Add 1 to cycle.

18. Once $\mathrm{FAB}$ and $\mathrm{BAB}$ have established route path between source to destinations and data packets are send along the same path. The pheromone track value is strengthened means path is shortest path between these two nodes.

19. Repeat above cycles (4-18) until cycle reaches a predefined Maximum Cycle Number (MCN)

\section{Simulation OUTCOMES}

This part explains the experimentation tools setup and parameters utilized in simulation of a MANET network. Ubuntu 11 is utilized like the operating system because it is an easy to use that makes it simple to run. Network Simulation 2 (NS2.35) is utilized as simulation software that performs effortlessly over Ubuntu 11. In Table 2, we explain MANET parameters which are utilized in this simulation to calculate its functioning and contrast it with diverse protocols over a MANET network. In the simulation, we learn the relation between different MANET performance parameters regarding packets' size.

Table 2: Parameters Used in Simulation

\begin{tabular}{|l|l|}
\hline Parameter & Value \\
\hline Operating system & Ubuntu 11 \\
\hline NS2-2 version & 2.35 \\
\hline Channel type & Wireless Channel \\
\hline Amount of nodes & 100 \\
\hline Speed & $3,5,7,10,20,25$ \\
\hline Data type & UDP \\
\hline Simulation time & 160 seconds \\
\hline MAC protocol & MAC/802.11 \\
\hline Data packet size & $100,300,500,700,800,1000,1500$ and 2000 \\
\hline Area of simulation & $700 * 700$ \\
\hline Radio Propagation Model & Two ray ground \\
\hline Routing Protocol & AODV/DSR \\
\hline
\end{tabular}


The presented Hybrid Improved Artificial bee colony and Simulated annealing (HIASA) based Attack Detection (AD) algorithm is matched up with the previous Artificial Bee Colony(ABC), Memetic Artificial Bee Colony (MABC) algorithm [35] of safe route is gauged with reference to Packet Delivery Ratio(PDR), Throughput, End-to-End delay (E2E), routing efficiency and Routing Overhead (RO) etc. The performance and outcomes of the routing algorithm as follows:

\section{Throughput}

The throughput is known as the amount of bytes send out or received for every second. The throughput is indicated by $\mathrm{T}$,

$$
\begin{gathered}
\text { Throughput }=\text { received node } / \text { simulation time } \\
\qquad T=\frac{\sum_{i=1}^{n} N_{i}^{r}}{\sum_{i=1}^{n} N_{i}^{s}} \times 100 \%
\end{gathered}
$$

Here, $N_{i}^{r}$ average receiving node for the ith application, $N_{i}^{s}=$ average sending node for the ith application, and $\mathrm{n}=$ amount of applications.

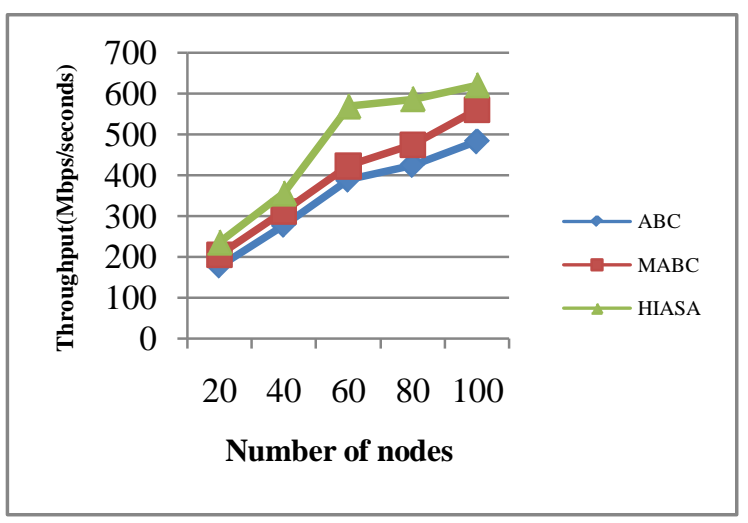

Figure 3(a): Throughput Comparison Outcomes of AODV Protocol

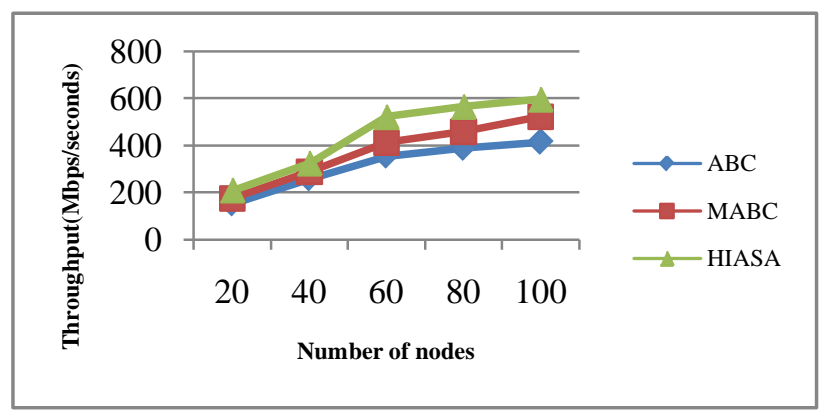

Figure 3(b): Throughput Comparison Outcomes of DSR Protocol

In Figure 3(a) and Figure 3(b) exhibits throughput evaluation outcomes of the attack discovery methods for instance $A B C, M A B C$ and HIASA. From these figures it brings to a close that the presented HIASA based AD algorithm enhance define throughput while matched up ith ABC, and MABC. Dependent upon the figure 3(a)\&(b), it is proved that HIASA do superior while the amount of nodes raises will bring about very steady path from source to destination. It proves that the amount of Mbps send out from source to destination has enlarges for HIASA based AD algorithm. The values of these methods are given in table 3 . In the table 3, illustrates that the presented HIASA based AD algorithm yields average throughput outcomes of 473.8 Mbps/seconds for AODV protocol, where the average throughput outcomes of MABC and ABC are $395.4 \mathrm{Mbps} /$ seconds and 350.6 Mbps/seconds correspondingly. In the same way the presented HIASA based AD algorithm yields average throughput outcomes of $444.6 \mathrm{Mbps} / \mathrm{seconds}$ for AODV protocol , where the average throughput outcomes of MABC and $\mathrm{ABC}$ are $371.4 \mathrm{Mbps} /$ seconds and $314.2 \mathrm{Mbps} /$ seconds correspondingly, it proves that the presented HIASA based $\mathrm{AD}$ algorithm does superior for both routing protocols

Table 3: Throughput Comparison Outcomes of AODV

\begin{tabular}{|c|c|c|c|c|c|c|}
\hline \multirow[t]{2}{*}{$\begin{array}{l}\text { No. of } \\
\text { nodes }\end{array}$} & \multicolumn{3}{|c|}{$\begin{array}{l}\text { Throughput(Mbps/Seconds)- } \\
\text { AODV }\end{array}$} & \multicolumn{3}{|c|}{$\begin{array}{l}\text { Throughput(Mbps/Seconds)- } \\
\text { DSR }\end{array}$} \\
\hline & ABC & MABC & HIASA & $\mathrm{ABC}$ & MABC & HIASA \\
\hline 20 & 178 & 205 & 236 & 152 & 175 & 210 \\
\hline 40 & 278 & 312 & 356 & 259 & 289 & 325 \\
\hline 60 & 389 & 423 & 569 & 356 & 412 & 523 \\
\hline 80 & 425 & 476 & 587 & 389 & 458 & 567 \\
\hline 100 & 483 & 561 & 621 & 415 & 523 & 598 \\
\hline Avg & 350.6 & 395.4 & 473.8 & 314.2 & 371.4 & 444.6 \\
\hline
\end{tabular}
Protocol

Packet Delivery Ratio(PDR)

It could be calculated as the proportion of the obtained packets by the destination nodes to the packets forwarded by the source node.

$\mathrm{PDR}=$ (amount of received packets / amount of sent packets) $* 100$

$$
T=\frac{\sum_{i=1}^{n}\left(N_{i}^{S}-N_{i}^{r}\right)}{\sum_{i=1}^{n} N_{i}^{S}} \times 100 \%
$$

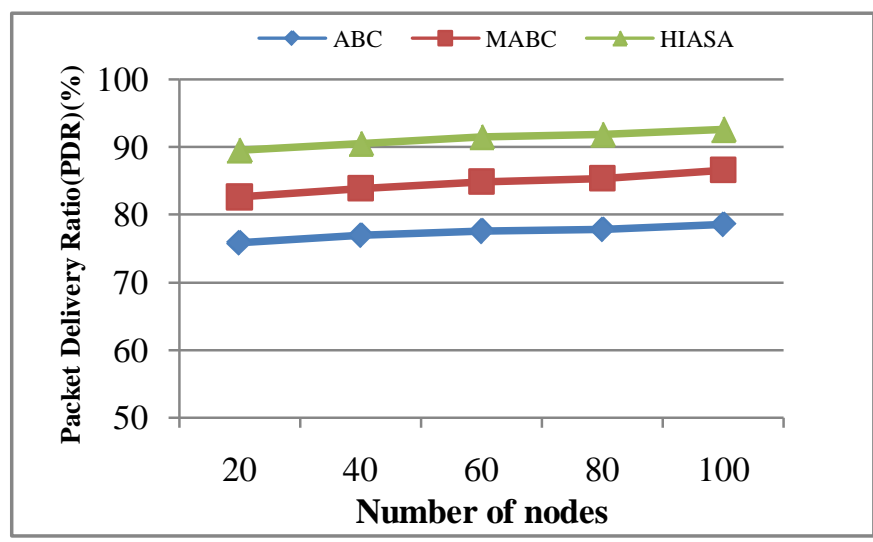

Figure 4(a): PDR Comparison Outcomes of AODV Protocol

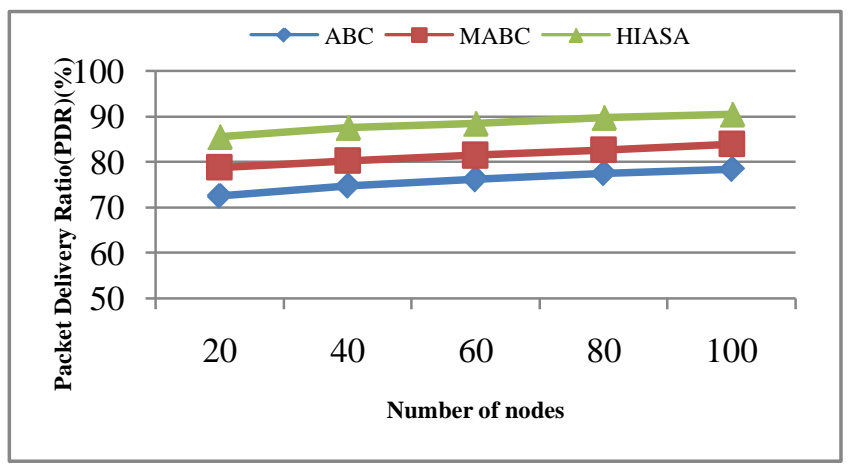

Figure 4(b): PDR Comparison Outcomes of DSR Protocol 
Figure 4(a) and (b) illustrates that packet delivery ratio of the presented HIASA based $\mathrm{AD}$ algorithm and previous enhancement methods. As a lot of attacks has been identified and those route are taken away from original routing table. AODV raise the PDR of presented system and somewhat reduces if the amount of nodes rises. It demonstrates that the amount of packets forwarded from source to destination has raises for HIASA based AD algorithm. The values of these methods are given in table 4 . In the table 4 , demonstrates that the presented HIASA based AD algorithm generates average PDR outcomes is $91.176 \%$ for AODV protocol, where the average PDR outcomes of MABC and ABC are $84.61 \%$ and $77.344 \%$ correspondingly. In the same way the presented HIASA based AD algorithm generates average PDR outcomes of $88.372 \%$ for AODV protocol, where the average PDR outcomes of MABC and $\mathrm{ABC}$ are $81.376 \%$ and $75.862 \%$ correspondingly, it proves that the presented HIASA based $\mathrm{AD}$ algorithm generates superior PDR outcomes for both AODV and DSR routing protocols

Table 4: PDR Comparison Outcomes of AODV Protocol

\begin{tabular}{|c|c|c|c|c|c|c|}
\hline \multirow[t]{2}{*}{ No. of nodes } & \multicolumn{3}{|c|}{$\begin{array}{l}\text { Packet Delivery Ratio(PDR) (\%)- } \\
\text { AODV }\end{array}$} & \multicolumn{3}{|c|}{$\begin{array}{l}\text { Packet Delivery Ratio(PDR) } \\
\text { (\%)-DSR }\end{array}$} \\
\hline & ABC & MABC & HIASA & $\mathrm{ABC}$ & MABC & HIASA \\
\hline 20 & 75.89 & 82.56 & 89.52 & 72.56 & 78.75 & 85.62 \\
\hline 40 & 76.93 & 83.87 & 90.51 & 74.76 & 80.25 & 87.53 \\
\hline 60 & 77.52 & 84.79 & 91.45 & 76.14 & 81.43 & 88.46 \\
\hline 80 & 77.86 & 85.31 & 91.87 & 77.48 & 82.57 & 89.73 \\
\hline 100 & 78.52 & 86.52 & 92.53 & 78.37 & 83.88 & 90.52 \\
\hline Avg & 77.344 & 84.61 & 91.176 & 75.862 & 81.376 & 88.372 \\
\hline
\end{tabular}

\section{Dropped Packets Ratio}

It could be calculated as the proportion of the number of packets that transmitted by the source node and be unsuccessful to arrive at to the destination node

Dropped packets $=$ sent packets - received packets

$$
T=\sum_{i=1}^{n}\left(N_{i}^{s}-N_{i}^{r}\right)-\sum_{i=1}^{n} N_{i}^{s}
$$

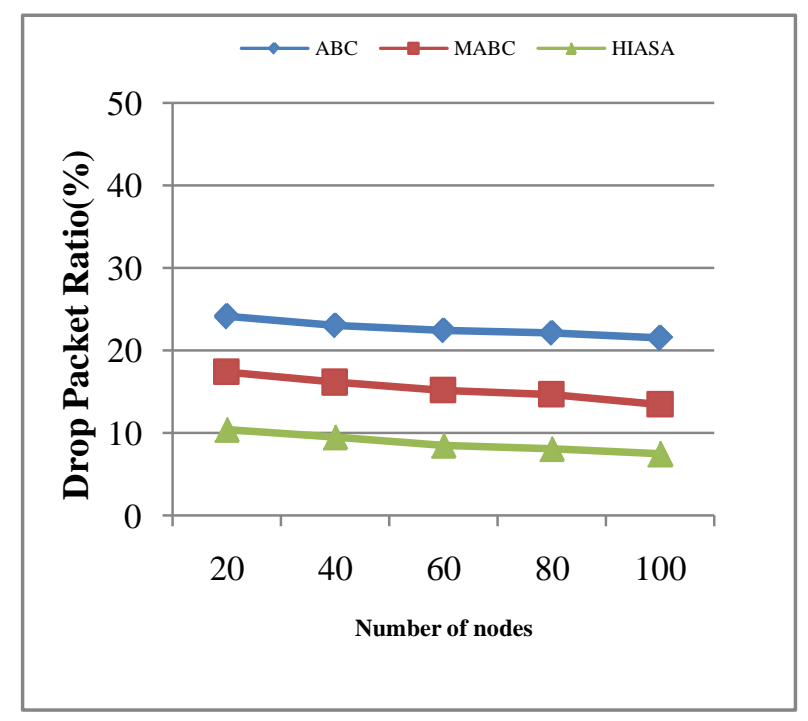

Figure 5(a): Drop Packets Ratio Comparison Outcomes of AODV Protocol

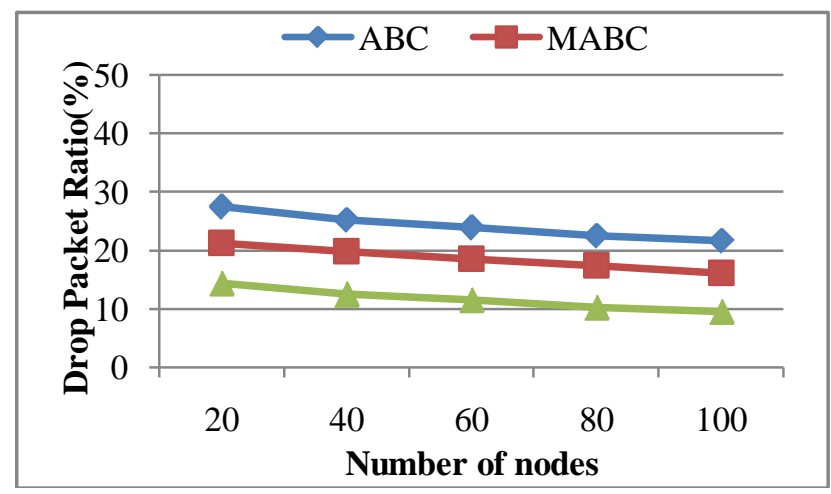

Figure 5(b): Drop Packets Ratio Comparison Outcomes of DSR Protocol

Figure 5(a) and (b) illustrates that drop packets ratio of the presented HIASA based AD algorithm and previous enhancement methods. From the outcomes it proves that the presented HIASA based AD algorithm has less amount of dropped packets outcomes than the previous methods for both routing protocols, as amount of attacks identified in the presented work is superior. Those routes have been taken away from original routing table, thus decreases amount of dropped packets. It illustrates that the amount of packets transmitted from source to destination has been superior. The values of these methods are specified in table 5. In the table 5, illustrates that the presented HIASA based AD algorithm generates drop packets ratio outcomes is $7.47 \%$ for 100 amount of nodes in the AODV protocol, where the drop packets ratio outcomes of MABC and $\mathrm{ABC}$ are $13.48 \%$ and $21.48 \%$ correspondingly. In the same way the presented HIASA based AD algorithm generates average drop packets ratio outcomes of $9.48 \%$ for 100 amount of nodes in the, where the drop packets ratio of MABC and ABC are $16.12 \%$ and $21.63 \%$ correspondingly.

Table 5: Average Drop Packets Ratio Comparison Outcomes of AODV Protocol

\begin{tabular}{|l|l|l|l|l|l|l|}
\hline \multirow{2}{*}{ No. of nodes } & \multicolumn{3}{|l|}{ drop packets ratio (\%)-AODV } & \multicolumn{3}{l|}{ drop packets ratio (\%)-DSR } \\
\cline { 2 - 7 } & ABC & MABC & HIASA & ABC & MABC & HIASA \\
\hline 20 & 24.11 & 17.44 & 10.48 & 27.44 & 21.25 & 14.38 \\
\hline 40 & 23.07 & 16.13 & 9.49 & 25.24 & 19.75 & 12.47 \\
\hline 60 & 22.48 & 15.21 & 8.55 & 23.86 & 18.57 & 11.54 \\
\hline 80 & 22.14 & 14.69 & 8.13 & 22.52 & 17.43 & 10.27 \\
\hline 100 & 21.48 & 13.48 & 7.47 & 21.63 & 16.12 & 9.48 \\
\hline
\end{tabular}

End-to-End delay (E2E)

It signifies the time needed to transmit the packet from the source node to the destination node.

E-2-E delay [packetid] = received time [packetid]- sent time

The average E2E could be computed by summing up the time consumed by all obtained packets divided by its totality amounts

$$
D=\frac{\sum_{i=1}^{n} d_{i}}{n}
$$

Here, $d_{i}=$ average end to end delay of node of $\mathrm{i}^{\text {th }}$ application and $\mathrm{n}=$ amount of application. 


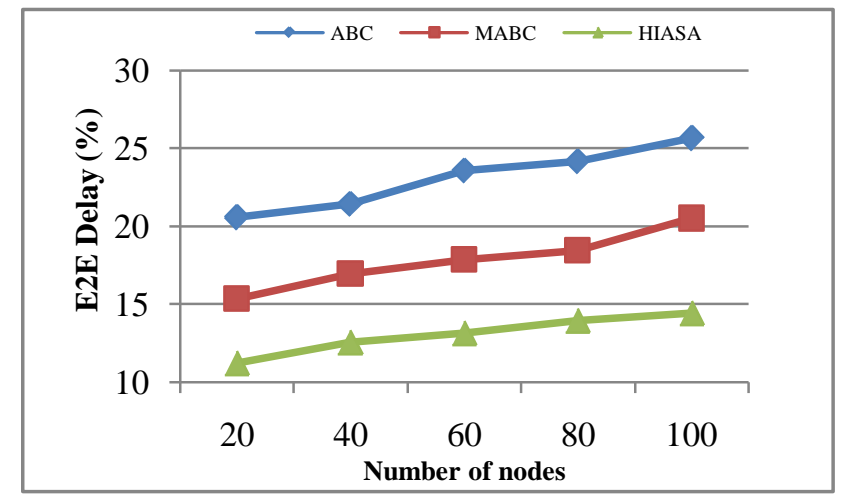

Figure 6(a): End-to-End delay (E2E) Comparison Outcomes of AODV Protocol

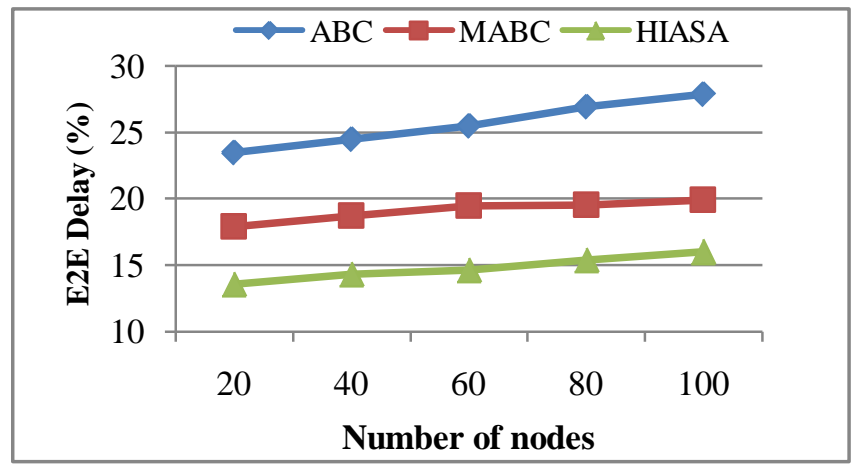

Figure 6(b). End-to-End Delay (E2E) Comparison Outcomes of DSR Protocol

Figure 6(a) and (b) illustrates that the E2E delay performance comparison outcomes of presented HIASA based $\mathrm{AD}$ algorithm and previous enhancement methods. From the outcomes it proves that the presented HIASA based AD algorithm has lesser E2E when compared to previous methods for both routing protocols. It illustrates that the amount of packets transmitted from source to destination has been superior. The values of these methods are specified in table 6 . In the table 6, illustrates that the presented HIASA based AD algorithm generates average E2E delay is $13.084 \%$ for AODV protocol, where the average E2E delay outcomes of MABC and $\mathrm{ABC}$ are $17.836 \%$ and $23.07 \%$ correspondingly .In the same way the presented HIASA based AD algorithm generates average E2E delay outcomes is $14.766 \%$, where the average E2E delay outcomes of MABC and ABC are $19.098 \%$ and $25.656 \%$ correspondingly.

Table 6: Average E2E Delay Comparison Outcomes of AODV Protocol

\begin{tabular}{|l|l|l|l|l|l|l|}
\hline \multirow{2}{*}{ No. of nodes } & \multicolumn{3}{|l|}{ E2E Delay (\%)-AODV } & \multicolumn{3}{l|}{ E2E Delay (\%)-DSR } \\
\cline { 2 - 7 } & ABC & MABC & HIASA & ABC & MABC & HIASA \\
\hline 20 & 20.56 & 15.36 & 11.25 & 23.48 & 17.87 & 13.54 \\
\hline 40 & 21.43 & 16.98 & 12.58 & 24.51 & 18.71 & 14.31 \\
\hline 60 & 23.58 & 17.87 & 13.17 & 25.48 & 19.45 & 14.63 \\
\hline 80 & 24.15 & 18.46 & 13.95 & 26.96 & 19.53 & 15.37 \\
\hline 100 & 25.63 & 20.51 & 14.47 & 27.85 & 19.93 & 15.98 \\
\hline Avg & 23.07 & 17.836 & 13.084 & 25.656 & 19.098 & 14.766 \\
\hline
\end{tabular}


[7] T. Eissa, S.A. Razal and M.D. Asringadi, "Enhancing MANET security using secret public keys", International Conference on Future Networks, Pp.130-134, 2009.

[8] N. Kang, E.M. Shakshuki and T.R. Sheltami, "Detecting forged Acknowledged in MANETs", International Conference on Advance Information Networking and Applications, Pp. 488-494, 2011.

[9] H. Martin and P. Deniele, "Routing in ad hoc networks: A case for long Hops”, IEEE Communications Magazine, Pp. 93-101, 2005.

[10] V. Singla and P. Kakkar, "Traffic Pattern based performance comparison of Reactive and Proactive protocols of Mobile Ad-hoc Networks", International Journal of Computer Applications, Vol. 5, No. 10, Pp.16-20, 2010.

[11] B. Renu and T. Pranavi, "Routing protocols in mobile ad-hoc network: a review", International Conference on Heterogeneous Networking for Quality, Reliability, Security and Robustness, Pp. 52-60, 2013.

[12] J. Krause, G.D. Ruxton and S. Krause, "Swarm intelligence in animals and humans", Trends in ecology \& evolution, Vol. 25, No. 1, Pp.28-34, 2010.

[13] X.S. Yang, Z. Cui, R. Xiao, A.H. Gandomi and M. Karamanoglu, "Swarm intelligence and bio-inspired computation: theory and applications”, Newnes, 2013.

[14] P.N. Raj and P.B. Swadas, "Dpraodv: A dyanamic learning system against blackhole attack in AODV based manet”, arXiv preprint arXiv:0909.2371, 2009.

[15] P.C. Tsou, J.M. Chang, Y.H. Lin, H.C. Chao and J.L. Chen, "Developing a BDSR scheme to avoid black hole attack based on proactive and reactive architecture in MANETs", $13^{\text {th }}$ International Conference on Advanced Communication Technology (ICACT).

[16] W. Wang, B. Bhargava and M. Linderman, "Defending against collaborative packet drop attacks on MANETs", $2^{\text {nd International }}$ Workshop on Dependable Network Computing and Mobile Systems (DNCMS), 2009.

[17] W. AlMobaideen, "SPDA: stability based partially disjoint AOMDV", European Journal of Scientific Presented, Vol. 27, No. 3, Pp.342-348, 2009.

[18] G. Di Caro, F. Ducatelle and L.M. Gambardella, "AntHocNet: an adaptive nature-inspired algorithm for routing in mobile ad hoc networks", European Transactions on Telecommunications, Vol. 16, No. 5, Pp. 443-455, 2005.

[19] Sh. Rahmatizadeh, H. Shah-Hosseini and H. Torkaman, "The Ant-Bee Routing Algorithm: A New Agent Based Nature-Inspired Routing Algorithm”, Journal of Applied Sciences, Vol. 9, No. 5, Pp. 983-987, 2009.

[20] N. Mazhar and M. Farooq, "Vulnerability analysis and security framework (BeeSec) for nature inspired MANET routing protocols", Proceedings of the $9^{\text {th }}$ annual conference on Genetic and evolutionary computation, Pp. 102-109, 2007.

[21] N. Mazhar and M. Farooq, "A sense of danger: dendritic cells inspired artificial immune system for MANET security”, Proceedings of the $10^{\text {th }}$ annual conference on Genetic and evolutionary computation, Pp. 63-70, 2008.

[22] F. Barani and M. Abadi, "BeeID: intrusion detection in AODV-based MANETs using artificial bee colony and negative selection methods", The ISC international journal of information security, Vol. 4, No. 1, Pp. 25-39, 2015.

[23] Vijay Anand and Jayakumar, “A Secure Routing Protocol to Mitigate Blackhole Attack in MANET using Artificial Bee Colony Enhancement”, Australian Journal of Basic and Applied Sciences, Vol. 9, No. 10, Pp. 31-38, 2015.

[24] D.B. Johnson, D.A. Maltz and J. Broch, "DSR: the dynamic source routing protocol for multi-hop wireless ad hoc networks", Ad hoc networking, pp.1-25, 2006.

[25] M.K. Marina and S.R. Das, "Ad hoc on-demand multipath distance vector routing", Wireless communications and mobile computing, Vol. 6, No. 7, Pp. 969-988, 2006.

[26] A. Vangili and K. Thangadurai, "Detection of black hole attack in mobile ad-hoc networks using ant colony enhancement-simulation analysis”, Indian Journal of Science and Technology, Vol. 8, No. 13, Pp.1-10, 2015.

[27] Y.A. Huang, W. Fan, W. Lee and P.S. Yu, "Cross-feature analysis for detecting ad-hoc routing anomalies”, Proceedings $23^{\text {rd }}$ International Conference on Distributed Computing Systems, Pp. 478-487, 2003.

[28] P. Ning and K. Sun, "How to misuse AODV: A case study of insider attacks against mobile ad-hoc routing protocols", Proceedings of the $4^{\text {th }}$ Annual IEEE Information Assurance Workshop, Pp. 60-67, 2003.
[29] C.Y. Tseng, P. Balasubramanyam, C. Ko, R. Limprasittiporn, J. Rowe, and K. N. Levitt, "A specification-based intrusion detection system for AODV”, ACM Workshop on Security of Ad Hoc and Sensor Networks (SASN'), 2003.

[30] E. Aarts, J. Korst and W. Michiels, "Simulated annealing", Search methodologies, Pp. 265-285, 2014.

[31] H.R. Tizhoosh, "Opposition-based learning: a new scheme for machine intelligence", International conference on Computational intelligence for modelling, control and automation, and international conference on intelligent agents, web technologies and internet commerce, Vol. 1, Pp. 695-701, 2005.

[32] M. Ventresca and H.R. Tizhoosh, "Improving the convergence of backpropagation by opposite transfer functions", International Joint Conference on Neural Networks, Pp. 4777-4784, 2006.

[33] S. Rahnamayan, H.R. Tizhoosh and M.M. Salama, "Opposition-based differential evolution”, IEEE Transactions on Evolutionary computation, Vol. 12, No. 1, Pp. 64-79, 2008.

[34] B. Alatas, "Chaotic bee colony methods for global numerical enhancement”, Expert Syst. Appl., Vol. 37, Pp. 5682-5687, 2010.

[35] F. Barani and A. Barani, "Dynamic intrusion detection in AODV-based MANETs using memetic artificial bee colony algorithm”, $22^{\text {nd }}$ Iranian Conference on Electrical Engineering (ICEE), Pp. 1040-1046, 2014. 